\title{
A potential-dependent Thiele modulus to quantify the effectiveness of porous electrocatalysts
}

Charles Tai-Chieh Wan*,1,2, Katharine V. Greco*,1,2, Amira Alazmi ${ }^{2,3}$, Robert M. Darling*,4, Yet-Ming Chiang*,1,5, and Fikile R. Brushett*,1,3,z

1 Joint Center for Energy Storage Research, Massachusetts Institute of Technology, 77 Massachusetts Avenue, Cambridge, Massachusetts 02139, United States

2 Department of Chemical Engineering, Massachusetts Institute of Technology, 77 Massachusetts Avenue, Cambridge, Massachusetts 02139, United States

3 Department of Chemistry, University Colleges at Nairiyah, University of Hafr Albatin (UHB), Nairiyah 31981, Saudi Arabia

4 Raytheon Technologies Research Center, East Hartford, Connecticut 06108, United States

5 Department of Materials Science and Engineering, Massachusetts Institute of Technology, 77 Massachusetts Avenue, Cambridge, Massachusetts 02139, United States

* Electrochemical Society Member

z Corresponding Author Email Address: brushett@mit.edu

\section{Abstract}

Electrochemical reactors often employ high surface area electrocatalysts to accelerate volumetric reaction rates and increase productivity. While electrocatalysts can alleviate kinetic overpotentials, diffusional resistances at the pore-scale often prevent full catalyst utilization. The effect of intraparticle diffusion on the overall reaction rate can be quantified through an effectiveness factor expression governed by the Thiele modulus parameter. This analytical approach is integral to the development of catalytic structures for thermochemical processes and has previously been extended to electrochemical processes by accounting for the relationship between reaction kinetics and electrode overpotential. In this paper, we illustrate the method by deriving the expression for the potential-dependent Thiele modulus and using it to quantify the effectiveness factor for porous electrocatalytic structures. Specifically, we demonstrate the application of this mathematical framework to spherical microparticles as a function of applied overpotential across catalyst properties and reactant characteristics. The relative effects of kinetics and mass transport are related to overall reaction rates, revealing markedly lower catalyst utilization at increasing overpotential. Subsequently, we generalize the analysis to different catalyst shapes and provide guidance on the design of porous catalytic materials for use in electrochemical reactors.

Keywords: porous electrocatalyst, actitve area utilization, electrochemical engineering, Thiele modulus, effectiveness factor 


\section{Introduction}

Porous architectures have been strategically employed in heterogeneous catalytic processes to enhance volumetric reaction rates by increasing active site density. However, there exists a trade-off with mass-transport through the porous catalyst, which impacts the effective use of these catalyst sites. Classical approaches in chemical reactor design and engineering introduce an effectiveness factor to assess the ratio between the observed reaction rate and the reaction rate if the entire catalyst surface area been exposed to bulk concentration conditions. Intuitively, particle geometry (i.e., size and shape), catalyst surface reactivity, and intraparticle diffusion all impact the effectiveness factor. A dimensionless parameter, commonly termed the Thiele modulus, conveniently describes the relative balance between kinetic and mass transport resistances in the catalyst particle, quantifying the relation between catalyst particle size and activity, and was explored in Thiele's seminal 1939 paper. ${ }^{1-5}$ Specifically, the Thiele modulus is the ratio of a reaction rate to a diffusion rate. At large Thiele modulii, overall reaction rate is limited by diffusional resistance and the reaction is confined to the outer catalyst layers, whereas at small Thiele modulus values, the overall reaction rate is limited by the catalyst volume (i.e., total amount of accessible pore surface area). Generally, increases in this dimensionless group indicate masstransport-limited behavior and a more non-uniform concentration profile throughout the catalyst.

Historically, the Thiele modulus has been used to inform materials design for thermochemical processes, wherein relevant reactions are driven by temperature and pressure, supporting the development of effective porous media used in catalytic, separation, and adsorption technologies. ${ }^{6,7}$ This analytical approach can be extended to evaluate catalysts for electrochemical processes, but the potential-dependence of reaction rates requires a reformulation of the traditional effectiveness factor. Unlike temperature and pressure, which still impact reaction kinetics and 
mass transport in electrochemical systems, potential primarily impacts the reaction rate as it perturbs the free energy of the electrode-electrolyte interface, where the heterogeneous reactions occur, but does not typically impact bulk electrolyte properties. ${ }^{8}$

Traditional reactor engineering approaches have been extended in the development of effectiveness factors for electrochemical systems across multiple length scales. These approaches have notably been explored in room temperature fuel cells for modeling the activity of agglomerates, which can then be incorporated into macroscopic porous electrode models ${ }^{9}$ or multiscale simulations. ${ }^{10}$ Giner and Hunter first introduced the concept of flooded agglomerates to study Teflon-bonded gas diffusion electrodes, predicting the doubling of Tafel slopes in porous electrodes at higher current densities due to mass transport limitations from oxygen diffusion. ${ }^{11}$ Stonehart and Ross developed a framework to use effectiveness factors in porous layers to measure the kinetics of electrocatalysts with high rate constants as well as measure their surface poisoning by chemisorbed species, a demonstration which further accentuated the overlap between the fields of electrocatalysis and gas-phase heterogeneous catalysis. ${ }^{12}$ Iczkowski and Cutlip used the Thiele modulus for spherical agglomerates to determine the breakdown of overpotentials from ohmic and transport losses in fuel cell cathodes. ${ }^{13}$ The work of Perry et al. modeled mass transport behavior in both liquid-electrolyte and polymer-electrolyte fuel cell cathodes by using the rate expression for spherical agglomerates embedded into the catalyst layer and adding the effect of ionic mass transport in the electrolyte phase for the case of the liquid-electrolyte model. ${ }^{14}$ Further studies on modeling agglomerates and effectiveness factors in room-temperature fuel cells have refined and extended modeling and experimental approaches. ${ }^{15-23}$ In solid oxide fuel cells, Costagmagna et al. modeled the active functional catalyst layer using an effectiveness factor approach, where the modified Thiele modulus compared the ratio between rates of reaction and ionic charge transport 
through the active layer; ${ }^{24,24}$ subsequently, Shin and Nam incorporated Butler-Volmer kinetics into the rate expression, ${ }^{25}$ Baek et al. used this framework to hypothesize performance degradation, ${ }^{26}$ and Nam investigated the influence of various charge transfer coefficients. ${ }^{27}$ Recently, effectiveness factor analysis has also been utilized in gas diffusion electrodes for $\mathrm{CO}_{2}$ reduction to model agglomerates ${ }^{28}$ and as part of the development of an analytical model of the catalyst layer. ${ }^{29}$ Effectiveness factors have been applied to thermo-electrochemical cells, ${ }^{30}$ particulate bed electrodes, ${ }^{31,32}$ electrocatalysis in metal-organic frameworks, ${ }^{33}$ and in oxygen reduction reactions. ${ }^{34}$ The array of applications and incorporation across multiple modeling length scales highlights the ubiquity of the Thiele modulus / effectiveness factor approach.

Accordingly, understanding and quantifying the utilization of micrometric porous electrocatalysts of uniform composition employed to increase available surface area may be a beneficial tool to evaluate new catalytic materials in novel or established systems where this approach has remained unused. For example, recent efforts to improve the performance of redox flow batteries have included the deposition of high surface carbonaceous microparticles onto the surface of carbon-fiber bed electrodes. ${ }^{35-39}$ While some of the reported surface areas exceeding $500 \mathrm{~m}^{2} \mathrm{~g}^{-1}$ are $2-3$ orders of magnitude larger than the low surface area fibrous scaffolds they are deposited on $\left(\sim 0.1-10 \mathrm{~m}^{2} \mathrm{~g}^{-1}\right),{ }^{40}$ cell-level performance improvements are comparatively marginal. While a number of factors can impact flow cell performance, ${ }^{41,42}$ this discrepancy suggests incomplete catalyst utilization, or, specifically, that the benefits derived from increasing surface area in this fashion have an upper bound. More broadly, understanding the interplay between active surface area and diffusion is universally applicable and has utility in electrochemical engineering. The diverse portfolio of electrode configurations (e.g., volumetric matrixes, ${ }^{8,43,44}$ packed beds, ${ }^{31,32}$ slurry electrodes ${ }^{45,46}$ ) tailored to various objectives (e.g., removal 
of trace contaminants, ${ }^{47,48}$ electro-assisted selective adsorbent design, ${ }^{49}$ energy storage,${ }^{44,50}$ flow capacitors $^{51-53}$ ) motivate the general mathematical framework presented in this study. This general approach can be refined to specific electrochemical systems by appropriate selection of the reaction term and/or modeling domain.

Here, we present the potential-dependent Thiele modulus to quantify the effectiveness factor and utilization of porous electrocatalysts as functions of particle and reactant properties. We develop formulations to assess effectiveness factors as a function of applied potential using both Tafel and Butler-Volmer kinetics, and assuming one-dimensional reaction-diffusion through a porous sphere. We then outline design principles for electrocatalyst sizing based on desired utilization. Subsequently, we explore and quantify internal transport limitations arising from diffusional processes within the catalyst and external transport limitations arising from mass transfer from the bulk electrolyte to the outer surface of the catalyst. Finally, we extend the model to other common catalyst geometries using a shape factor analysis. While the reactant and catalyst conditions used in this work are based on an aqueous electrolyte at room temperature, the mathematical framework is generalizable and applicable to porous electrocatalysts across a range of conditions. 


\section{Model Development}

\subsection{Assumptions and formulation}

The model described herein considers the effectiveness factor in porous, spherical electrocatalysts (depicted in Figure 1). In this treatment, we contemplate a single-electron oxidation half-cell reaction (i.e., free electrons are treated as reactants or products) under isothermal conditions using full Butler-Volmer kinetics and the Tafel approximation. Alternative reaction orders and combinations are not considered in this treatment but can be incorporated by changing the reaction term, for which additional mathematical derivation may be needed. We assume dilute solution theory holds for describing ion transport properties. ${ }^{43}$ By convention, oxidative currents are positive, while reductive currents are negative. Three distinct concentration regions are considered, specifically the bulk region $\left(\mathrm{C}_{\text {bulk }}\right)$, the external boundary layer from the bulk to the surface of the catalyst $\left(\mathrm{C}_{\text {external }}\right)$, and the internal porous electrocatalyst $\left(\mathrm{C}_{\text {internal }}\right)$. The electrolyte solution is treated as a typical aqueous electrolyte consisting of a dissolved electroactive species with excess supporting salt. Several assumptions are made about the catalyst particle and its interaction with the surrounding electrolyte. Specifically, we assume that i) the electrolyte wets the entirety of the particle; ii) the physical properties of the particle (e.g., porosity and tortuosity) are spatially invariant and can be approximated by a single value; and iii) the species diffusion coefficient and rate constant remain at the same values throughout the particle. ${ }^{2,3,5}$ As such, we inherently assume that continuum behavior of the electrolyte holds throughout the catalyst and neglect non-idealities that may arise in confined spaces of nanometric dimensions, ${ }^{54-56}$ setting a lower bound on the particle and pore sizes that may be considered via this approach. We neglect ohmic losses across the particle as these contributions are usually minor in comparison to the charge transfer and mass transfer losses. More specifically, potential gradient in the solid phase is assumed to be negligible 
because the electronic conductivity in the catalyst solid phase is typically orders of magnitude greater than the solution-phase conductivity, ${ }^{57,58,20}$ though we refer the reader to other works that include gradients in the solid matrix in porous electrodes. ${ }^{9,17,59-61}$ Furthermore, if the particle is electronically conductive, only the electrolyte conductivity is significant, and if the cross-sectional area traversed by the reactants and supporting salt dissolved in the solvent is identical, the ohmic drop across the particle $(\Delta \eta(\mathrm{V}))$ can be estimated as Equation (1): ${ }^{12}$

$$
\Delta \eta \cong \frac{n F D_{e f f} \Delta C}{\kappa_{e f f}}
$$

where $n(-)$ is the number of electrons transferred, $F\left(\mathrm{C} \mathrm{mol}^{-1}\right)$ is the Faraday constant, $\Delta C\left(\mathrm{~mol} \mathrm{~cm}^{-3}\right)$ is the maximum possible concentration drop across the pellet, $\kappa_{\text {eff }}\left(\mathrm{S} \mathrm{cm}^{-1}\right)$ is the effective conductivity of the solution, and $D_{\text {eff }}\left(\mathrm{cm}^{2} \mathrm{~s}^{-1}\right)$ is the effective reactant diffusivity through the particle. The effective diffusivity in the catalyst pores is assumed to be $D_{e f f}=\varepsilon_{c a t} / \tau_{c a t} D_{b u l k}$, where $\varepsilon_{c a t}$ and $\tau_{c a t}$ are the porosity and tortuosity of the catalyst pellet, respectively, and $D_{b u l k}$ $\left(\mathrm{cm}^{2} \mathrm{~s}^{-1}\right)$ is the molecular diffusivity of the reactant species in the bulk electrolyte absent the influence of the catalyst particle. ${ }^{2}$ Based on this expression, the order of magnitude ohmic loss is $<<1 \mathrm{mV}$ for reasonable ranges of bulk active species electrolyte concentrations (ca. $5 \times 10^{-4} \mathrm{~mol}$ $\mathrm{cm}^{-3}$, or equivalently, $0.5 \mathrm{~mol} \mathrm{~L}^{-1}$ ), effective diffusion coefficients (ca. $10^{-6} \mathrm{~cm}^{2} \mathrm{~s}^{-1}$ ), and effective conductivities (ca. $0.2 \mathrm{~S} \mathrm{~cm}^{-1}$ ) observed in typical aqueous electrolytes. Thus, as the catalyst size is relatively small, the overpotential in the particle remains constant. ${ }^{12,17,62}$ Considerations for potential drop in the electrolyte phase on modeling domains of larger scale (i.e., catalyst layers and diffusion media) have been extensively covered in prior art. ${ }^{9,16,41,62,21}$ 


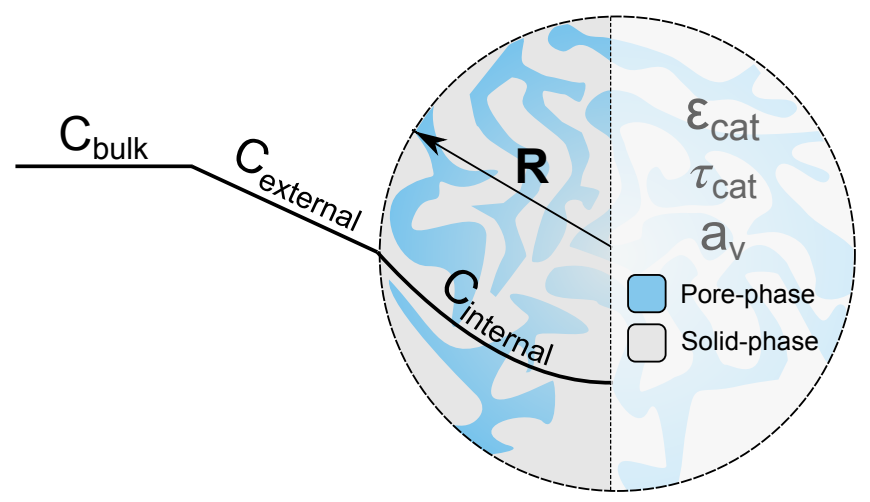

Figure 1 - Modeling domain. A two-dimensional representation of the reactant concentration in a spherical porous electrocatalyst. The concentration profile through the modeling domain can be divided into three distinct regions, namely the bulk $\left(\mathrm{C}_{\text {bulk }}\right)$, the external boundary layer $\left(\mathrm{C}_{\text {external }}\right)$, and the internal structure $\left(\mathrm{C}_{\text {internal }}\right)$. Important physical properties of the electrocatalyst, including bulk porosity $\left(\varepsilon_{c a t}\right)$, bulk tortuosity $\left(\tau_{c a t}\right)$, and volumetric surface area $\left(a_{v}\right)$, are listed on the schematic. The pore-phase and the solid-phase of the catalyst are represented by blue and gray, respectively.

\section{$3.2 \quad$ Tafel Kinetics}

First, consider a species A irreversibly oxidizing to species B in a single-electron half-cell reaction $(\mathrm{n}=1)$ as shown in Equation (2):

$$
A \rightarrow B+e^{-}
$$

The expression for the reaction rate assuming Tafel kinetics relating current to the applied overpotential for the oxidation reaction can be written as Equation (3):

$$
\bar{r}=\frac{i}{n F}=k_{f} c_{A}
$$


where $\bar{r}\left(\mathrm{~mol} \mathrm{~cm}^{-2} \mathrm{~s}^{-1}\right)$ is the reaction rate, $i\left(\mathrm{~mA} \mathrm{~cm}^{-2}\right)$ is the current density, $k_{f}\left(\mathrm{~cm} \mathrm{~s}^{-1}\right)$ is the potential-dependent heterogeneous forward rate constant, and $c_{A}\left(\mathrm{~mol} \mathrm{~cm}^{-3}\right)$ is the concentration of species A.

The electrochemical rate constant, $k_{f}$, can be written in terms of the standard heterogeneous rate constant, $k^{0}\left(\mathrm{~cm} \mathrm{~s}^{-1}\right)$ in Equation (4) as follows: ${ }^{63}$

$$
k_{f}=k^{0} \exp \left[\frac{\alpha_{f} n F \eta}{R T}\right]
$$

where $\alpha_{f}(-)$ is the forward charge-transfer coefficient, $R\left(\mathrm{~J} \mathrm{~mol}^{-1} \mathrm{~K}^{-1}\right)$ is the universal gas constant, $T(\mathrm{~K})$ is the absolute temperature, and $\eta(\mathrm{V})$ is the overpotential, defined here as $\eta=E-E_{f}^{0}$, where $E(\mathrm{~V})$ is the potential and $E_{f}^{0}(\mathrm{~V})$ is the formal potential.

The derivation of the effectiveness factor for a porous sphere that is a function of the Thiele modulus reflects traditional chemical engineering treatments, ${ }^{2,3}$ and, for completeness, is provided in Section 1 of the Supporting Information. The non-dimensionalized differential equation with boundary conditions of 1) reactant concentration at the surface of the sphere equal to the bulk concentration, $c_{A, b u l k}\left(\mathrm{~mol} \mathrm{~cm}{ }^{-3}\right)$, and 2) zero-flux at the center of the sphere can be written as

\section{Equation (5):}

$$
\frac{d^{2} \psi}{d \omega^{2}}+\frac{2}{\omega} \frac{d \psi}{d \omega}-\phi^{2} \psi=0 ; \quad \psi(\omega=1)=1 ; \quad \frac{d \psi}{d \omega}(\omega=0)=0
$$

The dimensionless concentration and dimensionless position are defined as $\psi=c_{A} / c_{A, b u l k}$ and $\omega=r / R_{p}$, respectively, where $r(\mathrm{~cm})$ is the coordinate radius, and $R_{p}(\mathrm{~cm})$ is the particle radius; 
the dimensionless number $\phi$ is the Thiele modulus, written for electrochemical rate constants with units of $\mathrm{cm} \mathrm{s}^{-1}$ as Equation (6):

$$
\phi=R_{p} \sqrt{\frac{a_{v} k_{f}}{D_{e f f}}}
$$

where $a_{v}\left(\mathrm{~cm}^{-1}\right)$ is the surface area to volume ratio, or volumetric surface area, of the catalyst particle. We note that the inclusion of terms in the differential equation indicates that the scaling of the kinetics and diffusion are on the same order of magnitude. The first boundary condition also implies rapid mass transfer through the boundary layer compared to mass transfer inside the sphere; this assumption is lifted in Section 4.2, where external transport limitations are explicitly treated.

The solution to Equation (5) that gives the dimensionless concentration profile is Equation (7):

$$
\psi=\frac{c_{A}}{c_{A, b u l k}}=\frac{\sinh (\phi \omega)}{\omega \sinh (\phi)}
$$

The effectiveness factor, $\mathrm{H}$, compares the observed reaction rate in the presence of diffusional transport limitations through the porous electrocatalyst $\left(\bar{r}_{o b s}\right)$ to the reaction rate in the presence of bulk electrolyte concentrations $\left(\bar{r}_{\max }\right)$. The relationship is given by Equation (8):

$$
\mathrm{H}=\frac{\bar{r}_{\text {obs }}}{\bar{r}_{\max }}=\frac{\int_{0}^{V_{p}} \bar{r}\left(c_{A}\right) d V}{V_{p} \bar{r}\left(c_{A, b u l k}\right)}
$$

where $V_{p}\left(\mathrm{~cm}^{3}\right)$ is the geometric volume of the particle. Substituting terms and rearranging leads to an expression of the effectiveness factor for any first-order reaction in a sphere, Equation (9): 


$$
\mathrm{H}=\frac{3}{\phi^{2}(\eta)}[\phi(\eta) \operatorname{coth}(\phi(\eta))-1]
$$

Introducing the potential-dependent heterogeneous rate constant, $k_{f}$, into Equation (6) yields a modified Thiele modulus, Equation (10):

$$
\phi^{2}(\eta)=R_{p}^{2} \frac{a_{v} k^{0}}{D_{e f f}} \exp \left(\frac{\alpha_{f} n F \eta}{R T}\right)
$$

where the equation can be separated into two dimensionless entities, an overpotential-independent parameter, $\gamma^{2}$, and an overpotential-dependent parameter, E, shown in Equation (11):

$$
\phi^{2}(\eta)=\gamma^{2} \mathrm{E}(\eta) ; \quad \gamma^{2}=R_{p}^{2} \frac{a_{v} k^{0}}{D_{e f f}} ; \quad \mathrm{E}(\eta)=\exp \left(\frac{\alpha_{f} n F \eta}{R T}\right)
$$

$\gamma^{2}$ is the conventional Thiele modulus squared without potential dependence, relating reaction kinetics to diffusion through the characteristic length of the particle. Note the above derivation is analogous for a reduction reaction. The formulation above is similar to the treatments that can be found in references ${ }^{14,17,20,62}$. The combination of Equation (9) and the dimensionless expression in Equation (11) result in a potential-dependent effectiveness factor for a porous sphere. Providing input values for the parameters in Equation (11) enables evaluation of catalyst utilization, as will be expanded upon in subsequent sections.

\section{$3.3 \quad$ Butler-Volmer Kinetics}

A similar derivation can be performed for a reversible single electron transfer $(n=1)$ described by Butler-Volmer kinetics, as shown in Equation (12):

$$
A \leftrightarrow B+e^{-}
$$


Assuming that the reactant concentration at the outer surface of the particle can be approximated by the reactant concentration in the bulk electrolyte (i.e., initially ignoring mass transport effects), the reaction-rate flux is described by Butler-Volmer kinetics with Equation (13):

$$
\bar{r}=\frac{i}{n F}=k_{f} c_{A}-k_{r} c_{B}
$$

where $k_{f}\left(\mathrm{~cm} \mathrm{~s}^{-1}\right)$ is the potential-dependent forward rate constant (here, anodic), and $k_{r}\left(\mathrm{~cm} \mathrm{~s}^{-1}\right)$ is the potential-dependent reverse rate constant (here, cathodic). Analogous to $k_{f}$, the electrochemical rate constant $k_{r}$ can be written in Equation (14) as:

$$
k_{r}=k^{0} \exp \left[\frac{-\alpha_{r} n F \eta}{R T}\right]
$$

Substitution of Equations (4) and (14) into Equation (13) leads to Equation (15):

$$
\bar{r}=k^{0} \exp \left[\frac{\alpha_{f} n F \eta}{R T}\right] c_{A}-k^{0} \exp \left[\frac{-\alpha_{r} n F \eta}{R T}\right] c_{B}
$$

With Butler-Volmer kinetics, the Thiele modulus now includes forward and backward reaction rates, and is defined as Equation (16):

$$
\phi^{2}(\eta)=R_{p}^{2} \frac{a_{v} k^{0}}{D_{e f f}}\left[\exp \left(\frac{\alpha_{f} n F \eta}{R T}\right)+\exp \left(\frac{-\alpha_{r} n F \eta}{R T}\right)\right]
$$

Similarly, the Thiele modulus can be deconvoluted into two dimensionless entities, shown in Equation (17):

$$
\phi^{2}(\eta)=\gamma^{2} E(\eta) ; \quad \gamma^{2}=R_{p}^{2} \frac{a_{v} k^{0}}{D_{\text {eff }}} ; \quad E(\eta)=\left[\exp \left(\frac{\alpha_{f} n F \eta}{R T}\right)+\exp \left(\frac{-\alpha_{r} n F \eta}{R T}\right)\right]
$$


The effectiveness factor for the Butler-Volmer reaction is identical to Equation (9) with the only difference being the use of Equation (16) as the Thiele modulus; the full derivation for the ButlerVolmer effectiveness factor and recovery of Equation (16) can be found in the Appendix. Computational analyses were executed in $\mathrm{MATLAB}^{\circledR}$ R2019a on a Dell Latitude 7280 laptop computer with an Intel ${ }^{\circledR}$ Core ${ }^{\mathrm{TM}} \mathrm{i} 7-6600 \mathrm{U}$ processor (2 Cores, $2.60 \mathrm{GHz}$ ) and random-access memory of $16 \mathrm{~GB}$. All the calculations for multiples geometries (e.g., spherical, slab, annulus; vide infra) and generated plots were run on a single script that required ca. $1.5-2$ min to execute.

\subsection{Model Output}

We next examine use of the model for fixed parameters across varying applied oxidative overpotential. Figure 2a shows the dimensionless concentration as a function of dimensionless length at different overpotentials for the full Butler-Volmer (dashed lines) kinetics and the Tafel approximation (solid lines) for a fixed $\gamma^{2}=1$. The transfer coefficient is assumed to be 0.5 for both the forward and reverse reactions, with a single-electron transfer. The modified, potentialdependent Thiele modulus for various values of $\gamma^{2}$ is shown as a function of overpotential in Figure 2b, used to calculate the effectiveness factor in Figure 2c. As expected, an apparent deviation at low overpotential between the kinetic treatments disappears at greater applied potentials as the Tafel approximation becomes increasingly accurate. The deviation between the effectiveness factor using Tafel and Butler-Volmer kinetics is initially exacerbated at low overpotential as $\gamma^{2}$ increases; however, the difference between effectiveness factors becomes $<$ $1 \%$ at ca. $100 \mathrm{mV}$ overpotential, which is observable in Figure 2c. Because the model outputs for Tafel and Butler-Volmer kinetics are fairly similar, for simplicity, Tafel kinetics are assumed for the remainder of this study. 

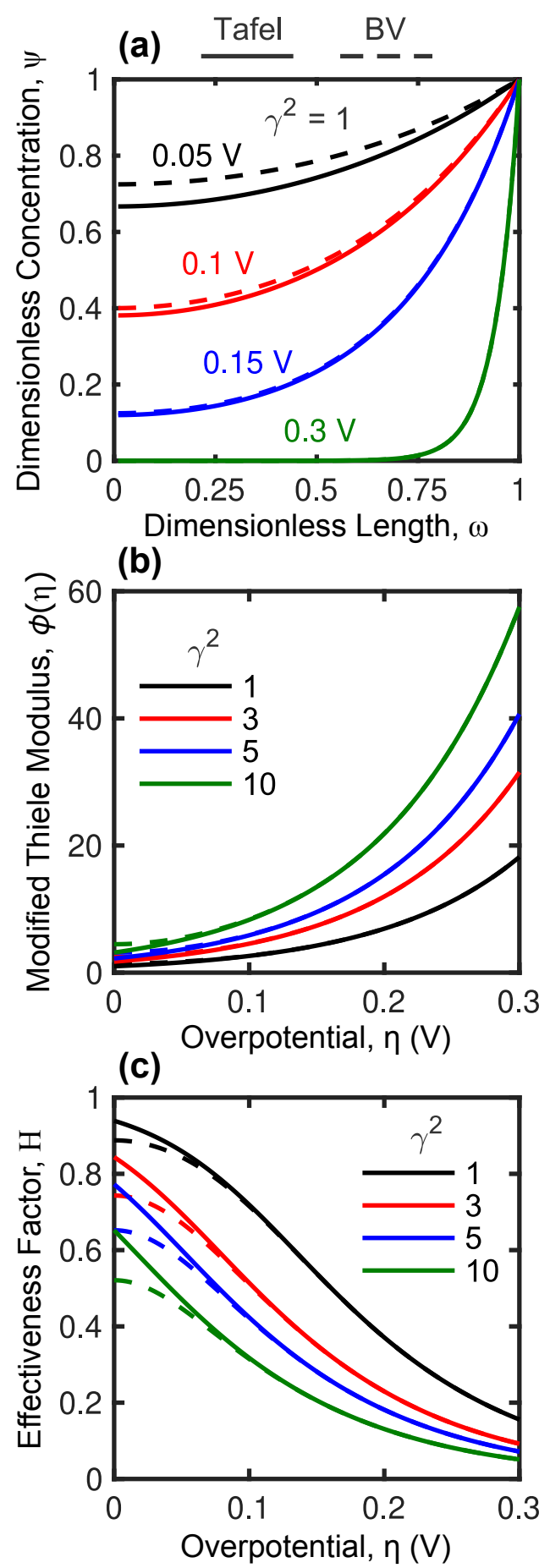

Figure 2 - Effectiveness factor through spherical porous electrocatalysts. (a) Dimensionless concentration as a function of dimensionless length for various oxidative overpotentials. (b) Modified Thiele modulus and (c) effectiveness factor as a function of overpotential for different $\gamma^{2}$ values (zero-potential Thiele modulus). Solid lines are for Tafel kinetics, while dashed lines indicate Butler-Volmer (BV) kinetics. 


\section{Results and Discussion}

In the following section, we apply the framework developed in Section $\mathbf{3}$ to size catalyst particles and generate polarization curves. We consider two regimes where transport effects take place ${ }^{5}$ (also depicted in Figure 1). Internal transport limitations arise when the reaction rate greatly exceeds the rate of reactant diffusion through the pore space of the catalyst particle. External transport limitations result as the reactant diffuses from the bulk to the catalyst surface through the boundary layer surrounding the particle. In Section 4.1, we consider solely internal transport limitations; in doing so, we implicitly assume rapid external mass transport (i.e., external transport limitations have a negligible effect). In Section 4.2, the assumption for rapid external mass transport is lifted, and both internal and external transport limitations are assessed.

\subsection{Internal Transport Limitations}

We now use the effectiveness factor derived in Section 2 to estimate the interfacial current density, $i$, in the presence of internal transport limitations, shown in Equation (18):

$$
i=\mathrm{H} n F k_{f} c_{A, b u l k}
$$

where $\mathrm{H}$ is the effectiveness factor. Substituting for $k_{f}$ and rearranging, we arrive at an expression for dimensionless internal current density, Equation (19):

$$
\frac{i}{n F k^{0} c_{A, b u l k}}=\mathrm{H} \exp \left(\frac{\alpha_{f} n F \eta}{R T}\right)
$$

The dimensionless current density increases exponentially with respect to overpotential. In the ideal scenario, $\mathrm{H}$ is 1 . The presence of internal transport limitations, however, reduces the dimensionless current density, lowering catalyst utilization. 
The relation between current density and effectiveness factor can be used to size catalysts to match a desired utilization efficiency, as shown in Figure 3. Lines in this figure represent the upper bound of particle radius for a given desired particle-specific current density for fixed effectiveness factors and volumetric surface areas. As particle radius increases, the efficiency (i.e., effectiveness factor) decreases; thus, the current density must be lowered to attain the desired efficiency. Similarly, as the volumetric surface area increases for fixed desired efficiency, the maximum allowable particle radius decreases accordingly. To generate Figure 3, fixed $D_{\text {eff }}=10^{-6} \mathrm{~cm}^{2} \mathrm{~s}^{-1}$ and $c_{A, b u l k}=5 \times 10^{-4} \mathrm{~mol} \mathrm{~cm}^{-3}$ (also $0.5 \mathrm{~mol} \mathrm{~L}^{-1}$ ) were selected as representative diffusivities and reactant concentrations. ${ }^{54,58,64}$ Ultimately, more precise values will depend on the specific electrolyte system (i.e., electrolyte composition, reactant concentration) and catalyst properties (i.e., tortuosity, porosity), which are combined into the effective diffusivity shown here. 


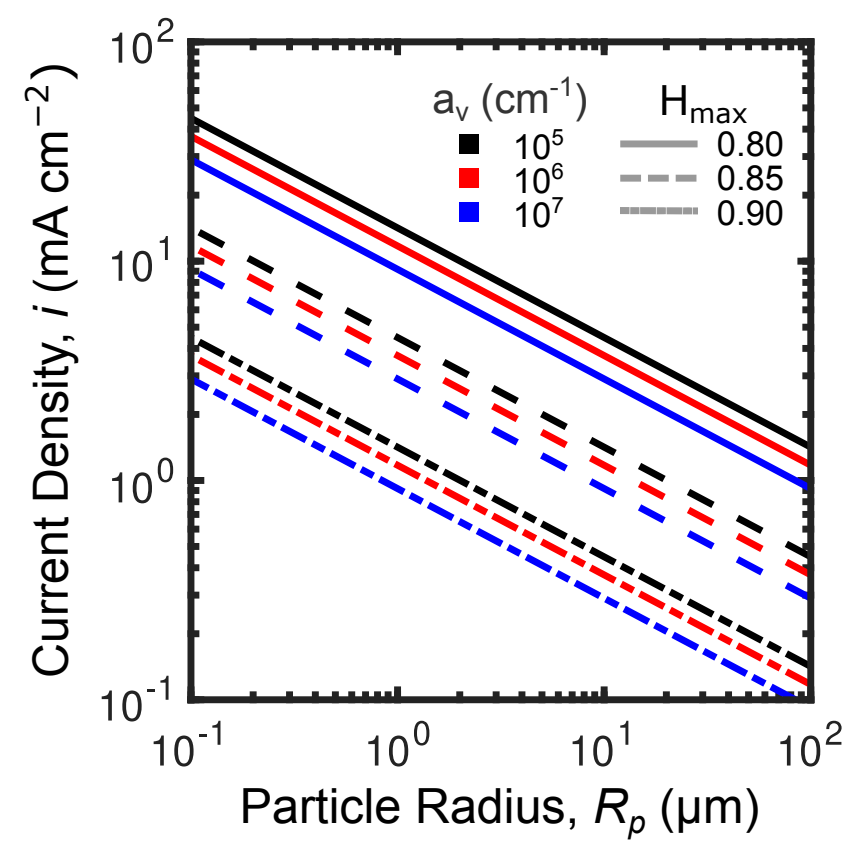

Figure 3 - Utilization and catalyst sizes. Particle-specific current density as a function of particle radius for the desired effectiveness factor $\left(\mathrm{H}_{\max }\right)$ and fixed volumetric surface areas $\left(a_{v}\right)$. $D_{\text {eff }}=10^{-6} \mathrm{~cm}^{2} \mathrm{~s}^{-1}$ and $c_{A, b u l k}=5 \times 10^{-4} \mathrm{~mol} \mathrm{~cm}^{-3}$ were held constant. The volumetric surface area is denoted by the line color, while the effectiveness factor is denoted by the line style. As particle volumetric surface area increases, the maximum allowable particle size at a given current density decreases. Similarly, increasing the utilization efficiency results in a lower maximum particle size.

The influence of the effectiveness factor on catalyst performance can also be visualized using polarization curves. Figure 4a shows the dimensionless internal current density as a function of overpotential according to Equation (19), with the ideal case (no losses, $H=1$ ) shown as the dotted black line, while the bold red, blue, and green curves represent the polarization curves for the three kinetic rate constants $\left(k^{0}\right)$ of increasing orders of magnitude $\left(10^{-5}, 10^{-4}\right.$, and $10^{-3} \mathrm{~cm} \mathrm{~s}^{-}$ 1 , respectively). Fixed values of $R_{p}=3 \mu \mathrm{m}, D_{\text {eff }}=10^{-6} \mathrm{~cm}^{2} \mathrm{~s}^{-1}$, and $a_{v}=10^{5} \mathrm{~cm}^{-1}$ were used based on experimentally-informed parameters. ${ }^{54,58,64}$ The volumetric surface area, $a_{v}$, is the product of the catalyst particle density and BET-derived surface area from nitrogen physisorption, 
based on approximate densities and surface area of porous carbon particles. ${ }^{65-67}$ The lightly shaded lines in between the bold lines represent intermediate kinetic rate constant values. Increments of $10^{-5} \mathrm{~cm} \mathrm{~s}^{-1}$ were used to transition from the red to blue curves, while increments of $10^{-4} \mathrm{~cm} \mathrm{~s}^{-1}$ were used to transition from the blue to green curves. We note that while the rate constant was varied in Figure 4, changing the particle size by the square root of the proportional change in the kinetic rate constant has an equivalent effect on the effectiveness factor. As the kinetic rate constant linearly increases, the dimensionless current density gradually consolidates. Figure $\mathbf{4 b}$ shows the corresponding effectiveness factors which evince severe limitations. The magnitude of the reduction in effectiveness is increasingly stark as the intrinsic kinetic rate constant increases, and intraparticle diffusion becomes limiting. The results indicate that even if the entire surface area of the catalyst particle were accessible for the electrochemical reaction, diminishing returns would be observed due to internal diffusional resistances. 


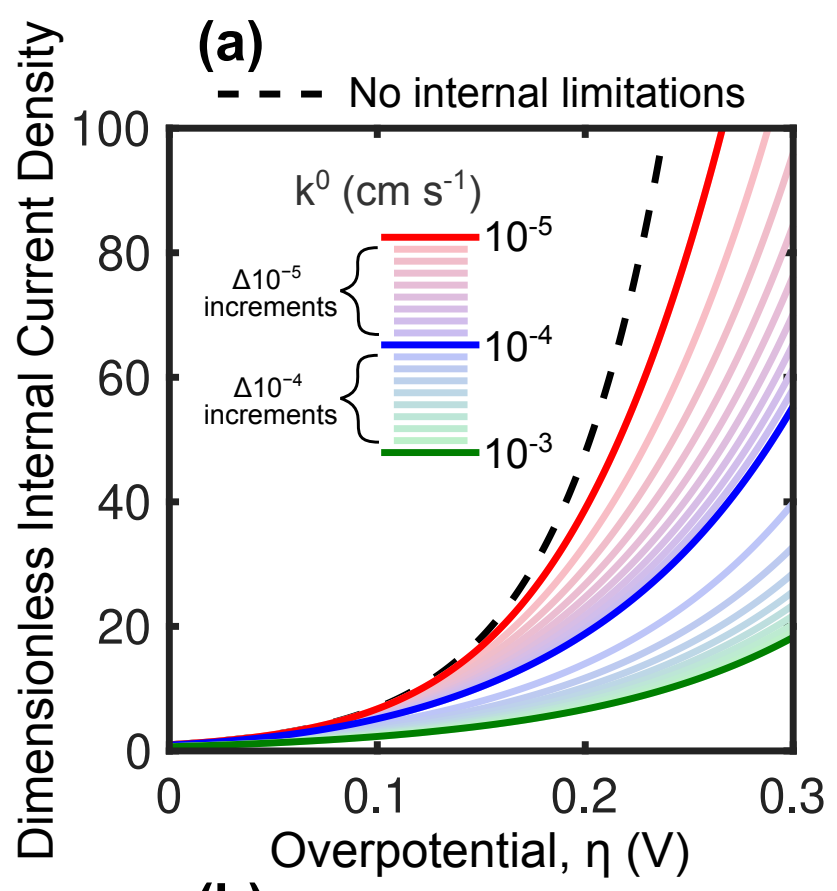

(b)

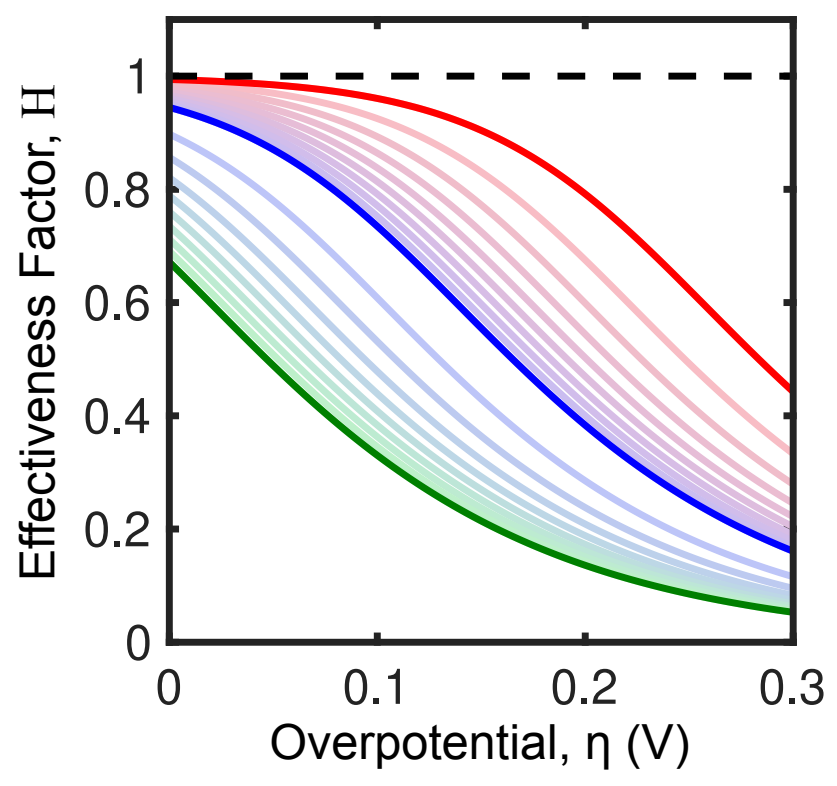

Figure 4 - Tafel plots for several kinetic rate constants at fixed diffusivity, volumetric surface area, and particle size. (a) Dimensionless internal current density as a function of overpotential. The black, dashed curve represents ideal polarization $(\mathrm{H}=1)$, while the solid red, blue, and green represent the three different kinetic rate constants of $10^{-5}, 10^{-4}$, and $10^{-3} \mathrm{~cm} \mathrm{~s}^{-1}$, respectively. The lightly shaded lines are increments in the rate constant, either $10^{-5}$ or $10^{-4} \mathrm{~cm} \mathrm{~s}^{-1}$ as indicated in the figure. (b) Effectiveness factor as a function of overpotential. The reaction rates follow the same coloring scheme as in (a). 


\subsection{External Transport Limitations}

To account for the effect of external mass transport from the bulk concentration to the outer surface of the catalyst particle, the total reaction rate on the interior of the catalyst particle is set equal to the reactant flux to the external surface (i.e., no accumulation on the catalyst surface boundary due to fouling, surface species absorption, etc.). Here, we assume that the particle is surrounded by electrolyte of uniform concentration without hindrance or obstruction from other catalyst particles, substrates, or other system components. ${ }^{2,3,5}$ Assuming that contributions from reactions along the outer surface of the particle are negligible compared to contributions from reactions throughout the volumetric internal surface area of the particle, ${ }^{2,3,5}$ and including the impact of the effectiveness factor on the reaction rate throughout the particle, results in Equation (20) for the flux balance:

$$
k_{m} a_{c}\left(c_{A, \text { bulk }}-c_{A, \text { surf }}\right)=\mathrm{H} k_{f} a_{v} c_{A . \text { surf }}
$$

where $k_{m}\left(\mathrm{~cm} \mathrm{~s}^{-1}\right)$ is the external mass transfer coefficient accounting for effects of diffusion and convection, and $a_{c}\left(\mathrm{~cm}^{-1}\right)$ is the catalyst shape factor, defined as the ratio of the particle external surface area to volume. Rearranging to solve for an expression for the surface concentration yields Equation (21):

$$
c_{A, \text { surf }}=\frac{k_{m} a_{c}}{k_{m} a_{c}+\mathrm{H} k_{f} a_{v}} c_{A, b u l k}
$$

Thus, the expression for the current density is now given by Equation (22):

$$
i=\mathrm{H} n F k_{f} c_{A, \text { surf }}=\frac{\mathrm{H} k_{f} k_{m} a_{c}}{k_{m} a_{c}+\mathrm{H} k_{f} a_{v}} n F c_{A, \text { bulk }}=\frac{\mathrm{H}}{1+\mathrm{H} \frac{k_{f} a_{v}}{k_{m} a_{c}}} n F k_{f} c_{A, \text { bulk }}
$$


Now, an expression for dimensionless current density including both internal and external transport with $\Omega$ as an overall effectiveness factor can be written as shown in Equation (23):

$$
\frac{i}{n F k^{0} c_{A, b u l k}}=\Omega \exp \left(\frac{\alpha_{f} n F \eta}{R T}\right) ; \quad \Omega=\frac{\mathrm{H}}{1+\mathrm{H} \frac{k_{f} a_{v}}{k_{m} a_{c}}}
$$

The effect of bulk mass transport is seen in Figure 5a, where the mass transfer coefficient is varied for fixed conventional Thiele modulus $\left(\gamma^{2}\right)$, and the current density at $\eta=0.3 \mathrm{~V}$ is shown on the ordinate. As the mass transport coefficient increases, losses due to reactant transport from the bulk to the catalyst surface decrease, and the dimensionless current density ultimately reaches a constant value which is dictated solely by internal diffusive losses through the catalyst particle. In other words, $\Omega \approx \mathrm{H}$ in the limit of a high mass transfer coefficient. As $\gamma^{2}$ increases, the limiting dimensionless current density has a lower value, which reflects poor catalyst particle utilization, as shown on a log-log base-10 scale with $\gamma^{2}$ in Figure 5 b. 
(a)
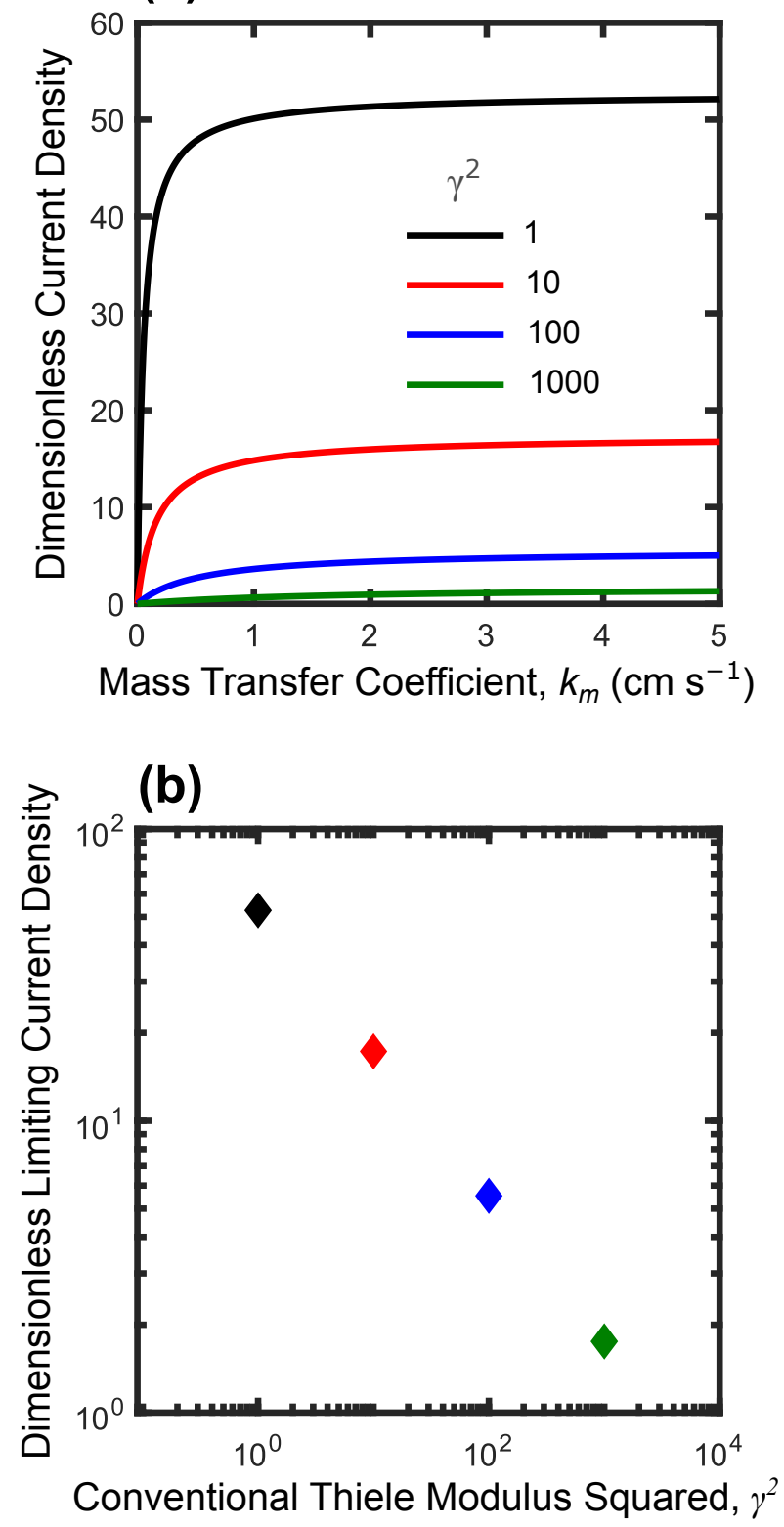

Figure 5 - Mass transfer dependence. (a) Dimensionless current density as a function of mass transfer coefficient for fixed $\gamma^{2}$ at $\eta=0.3 \mathrm{~V}$. (b) Dimensionless limiting current density extracted from (a) as a function of $\gamma^{2}$. 


\subsection{Extension of Model to General Geometries}

In practice, catalysts assume broad and irregular shapes. To accommodate the range of particle morphologies, we describe a simple transformation that can be used to extend the aforementioned framework to alternative geometries by using the catalyst volume $\left(V_{p}\right)$ to surface area $(S A)$ ratio as a characteristic length scale ( $L=V_{p} / S A$ ) in accordance with the seminal work of Aris ${ }^{68}$ Here, we use this facile strategy to analyze additional basic geometries (i.e., slab, cylinder, and annulus) as a demonstration for our model generalizability, although we posit that more advanced transformations with varying degrees of precision and complexity should also be compatible with the model formulation. Derivations for slab and cylinder catalysts are described in detail elsewhere; ${ }^{2-4,68}$ general equations for these geometries are presented in the Section 2 of the Supporting Information. A noteworthy geometry for fibrous catalysts is a porous reactive annulus that covers an impenetrable core. The porous reactive annulus can be realized via either additive or subtractive synthetic strategies. In additive processes, the porous layer is deposited or coated onto the fiber using particulate catalysts, ${ }^{69}$ conformal films, ${ }^{70}$ or porous mats: $;{ }^{71-73}$ whereas in subtractive processes, the porous layer is formed by surface roughening or fiber etching by thermal, ${ }^{74,75}$ chemical, ${ }^{76}$ or electrochemical ${ }^{77}$ means. 


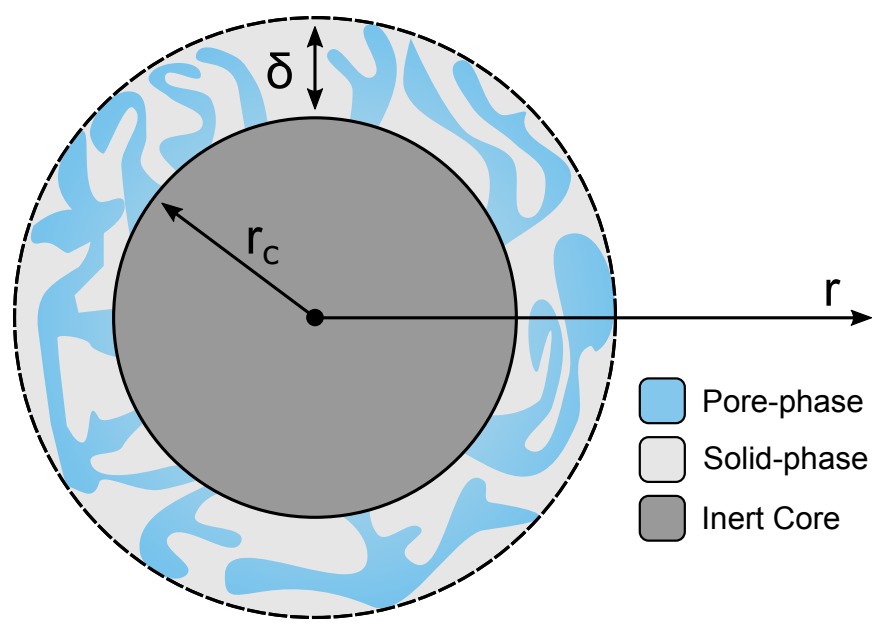

Figure 6 - Annulus geometry. Schema showing the annulus geometry and coordinates. $r_{c}$ is the radius of the non-reacting core, while $\delta$ is the shell thickness. The dark gray signifies the nonporous inert core. In the reactive porous phase, the blue and light gray represent the pore-phase and solid-phase of the catalyst, respectively.

Although the annular film morphology and activity varies based on the synthesis route, the mathematical framework described here broadly encompasses this category of materials. If the porous layer surrounds an impenetrable and nonporous core as a concentric shell, the governing equation for the $1 \mathrm{D}$ radial diffusion and reaction problem to solve for the dimensionless concentration profile becomes Equations (24) and (25): ${ }^{78,79}$

$$
\begin{gathered}
\frac{\partial^{2} \psi}{\partial \tilde{r}^{2}}+\frac{\xi}{\xi \tilde{r}+1} \frac{\partial \psi}{\partial \tilde{r}}-\phi^{2} \psi=0 ; \quad \frac{\partial \psi}{\partial \tilde{r}}(\tilde{r}=0)=0 ; \quad \psi(\tilde{r}=1)=1 \\
\psi=\frac{c_{A}}{c_{A, b u l k}} ; \quad \tilde{r}=\frac{r-r_{c}}{\delta} ; \quad \xi=\frac{\delta}{r_{c}} ; \quad \phi^{2}=\delta^{2} \frac{a_{v} k_{f}}{D_{e f f}}
\end{gathered}
$$

where $\tilde{r}(-)$ the dimensionless position, $r_{c}(\mathrm{~cm})$ the radius of the non-porous core, $\delta(\mathrm{cm})$ the thickness of the concentric shell, and $\xi(-)$ the ratio of the shell thickness to core thickness. A schema of the annulus with labeled variables is shown in Figure 6. The full derivation and 
numerical evaluation of Equation (24) are provided in Section 2 of the Supporting Information. The Thiele modulus in this form factor has a diffusion pathway across $\delta$. We can examine the limits of $\xi$ to ascertain behavior of the annulus at relatively large or small shell thicknesses. As $\xi \rightarrow 0$ (i.e, $\delta \ll r_{c}$ ), the effectiveness factor approaches that of an infinite slab. As $\xi \rightarrow \infty$ (i.e., $\left.\delta \gg r_{c}\right)$, the effectiveness factor approaches that of a cylinder.

The numerical solution for an annulus with $\xi=0.5$ as a function of overpotential is shown in Figure 7; effectiveness factors for an infinite slab, cylinder, and sphere corresponding to the same characteristic lengths are also shown for comparison. At low overpotentials - and consequently Thiele modulus — the effectiveness factor increases as the geometry transitions from the sphere to the slab. At high overpotentials - and consequently high Thiele modulus - the effectiveness factors collapse onto the same curve as diffusive limitations dominate and reactions are confined to the external layer, causing all geometries to behave effectively as slabs. Figure 7 may also be used to approximate irregular catalysts geometries using the $L=V_{p} / S A$ parameter. 


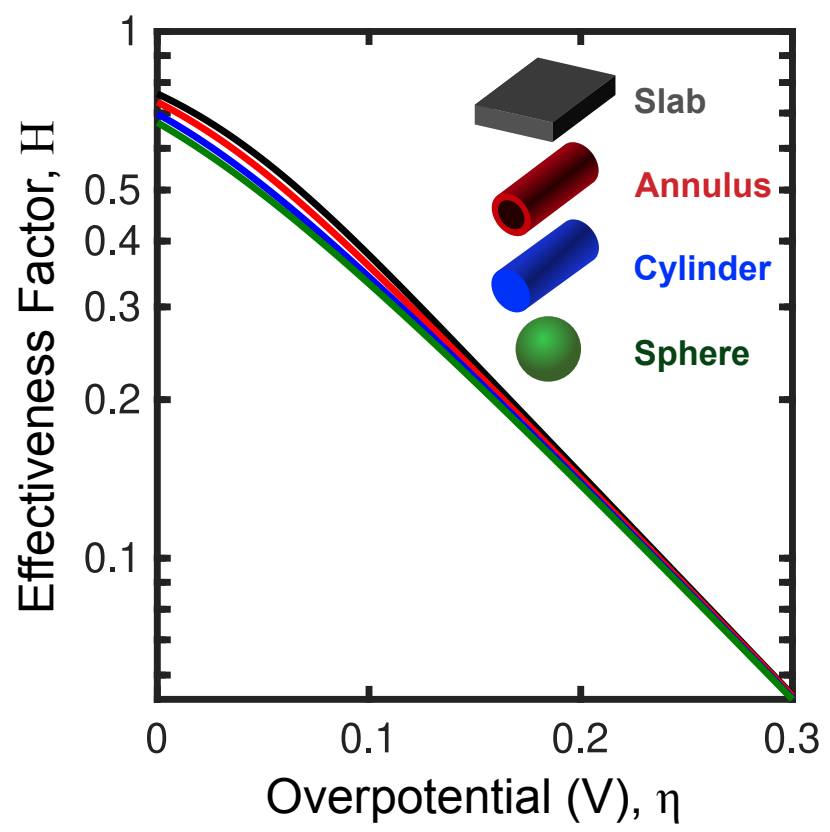

Figure 7 - Generalizability for various shape factors. Effectiveness factor as a function of overpotential calculated for a flat slab (grey), annulus (red), cylinder (blue), and sphere (green) based on their characteristic lengths. The curves collapse onto each other at larger overpotential as the reaction utilization shifts towards the outer edges of the catalyst independent of shape. 


\section{Conclusions}

Here we present a general strategy for quantifying potential-dependent effectiveness factors in porous electrocatalysts. The methodology extends the traditional Thiele modulus approach to include first-order electrochemical reactions, enabling investigation of the effect that diffusional losses have on net reaction rate in porous microstructures. Using this framework, we assess transport limitations both internally through a spherical particle - from the surface to the center and externally - from the bulk to the surface. We show how this approach may be used to target ideal catalyst sizes to achieve desired reaction efficiencies, assuming bulk-averaged morphological properties are known. Generally, the model suggests that low effectiveness factors manifest for larger driving forces, even at the relatively modest overpotentials examined $(\leq 0.3 \mathrm{~V})$. This work indicates that effective performance is not only dictated by the electrochemical reaction rate, but also by the comparative rate of mass transfer within the system; thus, the insights gained from the model findings highlight the existence of an upper-limit for effective surface area, and motivate the development of hierarchical structured electrocatalysts to mitigate diffusional pore-scale losses. To this end, a shape factor analysis is introduced to accommodate the breadth of catalyst geometries that may be used in electrochemical reactors. Future studies aiming to build on these methods should contemplate the role of electrolyte velocity and mass transfer coefficients on performance, the extension to other electrolyte systems and reaction sequences, along with the accurate estimation of the effective diffusion coefficient for liquid-phase reactions in highly tortuous microscale catalysts. 


\section{CRediT authorship contribution statement}

Charles Tai-Chieh Wan: Conceptualization, Methodology, Software, Validation, Formal Analysis, Investigation, Data Curation, Writing - Original Draft, Writing - Review \& Editing, Visualization. Katharine V. Greco: Conceptualization, Validation, Writing - Original Draft, Writing - Review \& Editing. Amira Alazmi: Conceptualization. Robert M. Darling: Conceptualization, Methodology, Writing - Review \& Editing. Yet-Ming Chiang: Resources. Fikile R. Brushett: Conceptualization, Writing - Original Draft, Writing - Review \& Editing, Project administration.

\section{Acknowledgements}

Research by C.T.W., Y.M.C., R.M.D., and F.R.B. was supported by the Joint Center for Energy Storage Research (JCESR), an Energy Innovation Hub funded by the U.S. Department of Energy, Office of Science, Basic Energy Sciences. A.A. acknowledges support from the Ibn Khaldun Fellowship for Saudi Arabian Women at MIT. C.T.W. and K.V.G. acknowledge graduate fellowships through the National Science Foundation Graduate Research Fellowship Program under Grant No. 1122374. Any opinions, findings, and conclusions or recommendations expressed in this material are those of the authors and do not necessarily reflect the views of the National Science Foundation. The authors also thank Alexis Fenton, Bertrand Neyhouse, and Kevin Tenny of the Brushett Group for their insightful discussions. 


\section{Appendix}

\section{Full Butler-Volmer Kinetics Derivation}

Here, we derive the Thiele modulus for a single-electron reaction described by Butler-Volmer kinetics. The reaction rate, $\bar{r}$, can be expressed as Equation (A1):

$$
\bar{r}=k_{f} c_{A}-k_{r} c_{B}
$$

which can be set equal to Equation (15) in the main text.

Performing a mass balance, we let the total concentration be defined as $C\left(\mathrm{~mol} \mathrm{~cm}^{-3}\right)$ as shown in Equation (A2):

$$
C=c_{A, b u l k}+c_{B, b u l k}=c_{A}+c_{B}
$$

Then, rearranging Equation (A2) and substituting into Equation (A1) results in Equation (A3):

$$
\bar{r}=k_{f} c_{A}-k_{r}\left(C-c_{A}\right)=k_{f} c_{A}+k_{r} c_{A}-k_{r} C=\left(k_{f}+k_{r}\right) c_{A}-k_{r} C
$$

Defining a dimensionless parameter $K$ as follows in Equation (A4):

$$
K=\frac{k_{f}}{k_{r}}=\frac{\exp \left[\frac{\alpha_{f} n F \eta}{R T}\right]}{\exp \left[\frac{-\alpha_{r} n F \eta}{R T}\right]}=\exp \left[\frac{\left(\alpha_{f}+\alpha_{r}\right) n F \eta}{R T}\right]
$$

Equation (A1) can be rewritten in terms of $k_{f}$ and concentration, giving Equations (A5) and (A6):

$$
\bar{r}=\left(k_{f}+k_{r}\right) c_{A}-k_{r} C=\left(k_{f}+\frac{k_{f}}{K}\right) c_{A}-\frac{k_{f}}{K} C
$$




$$
\bar{r}=k_{f}\left[1+\frac{1}{K}\right] c_{A}-\frac{k_{f}}{K} C=k_{f}\left[1+\frac{1}{K}\right]\left(c_{A}-\frac{C}{\left(1+\frac{1}{K}\right) K}\right)=k_{f}\left(1+\frac{1}{K}\right)\left(c_{A}-\frac{C}{K+1}\right)
$$

Performing a material balance on the porous sphere (mathematical treatment shown in Section 1 of the Supporting Information), and assuming that the reactant and product have equal effective diffusivities through the particle, we arrive at the following differential equation for a spherical particle, shown in Equation (A7):

$$
D_{e f f}\left[\frac{d^{2} c_{A}}{d r^{2}}+\frac{2}{r} \frac{d c_{A}}{d r}\right]-a_{v} k_{f}\left(1+\frac{1}{K}\right)\left(c_{A}-\frac{C}{K+1}\right)=0
$$

A solution requires rescaling the concentration appropriately to include the total concentration of the species, $\psi=c_{A}-\frac{C}{K+1}$. Non-dimensionalizing the length scale by $\omega=r / R_{p}$ results in the potential dependent Thiele modulus, Equation (A8):

$$
\phi^{2}=R_{p}^{2} \frac{a_{v} k_{f}\left(1+\frac{1}{K}\right)}{D_{\text {eff }}}=R_{p}^{2} \frac{a_{v} k^{0}}{D_{\text {eff }}}\left[\exp \left(\frac{\alpha_{f} n F \eta}{R T}\right)+\exp \left(\frac{-\alpha_{r} n F \eta}{R T}\right)\right]
$$

Which is equivalent to Equation (16) in the main text. Re-scaling of Equation (A7) and substitution of terms leads to Equation (A9):

$$
\frac{d^{2} \psi}{d \omega^{2}}+\frac{2}{\omega} \frac{d \psi}{d \omega}-\phi^{2} \psi=0 ; \quad \psi(\omega=1)=c_{A, b u l k}-\frac{C}{K+1} ; \quad \frac{d \psi}{d \omega}(\omega=0)=0
$$

which is the governing differential equation subject to the surface concentration and symmetric boundary conditions. The solution of Equation (A9) leads to a concentration profile for species

\section{A, Equation (A10):}




$$
c_{A}(\omega)=\frac{C}{K+1}+\left(\frac{c_{A, b u l k}-\frac{C}{K+1}}{\omega}\right) \frac{\sinh (\phi \omega)}{\sinh (\phi)}
$$

The effectiveness factor for a sphere with Butler-Volmer kinetics can be derived by relating the actual rate of reaction at the external particle surface to the total reaction throughout the entire particle volume if the concentration were the same as the outer surface. This leads to Equation (A11):

$$
\mathrm{H}=\frac{4 \pi R_{p}^{2} D_{\text {eff }} \frac{d c_{A}}{d r}}{r_{A, 0} V_{p}}
$$

Substituting for $V_{p}=\frac{4}{3} \pi R_{p}^{3}$, inserting the reaction rate, using the scaling for $\psi$ and modifying by $\omega$ results in Equation (A12):

$$
\mathrm{H}=\frac{4 \pi R_{p} D_{\text {eff }}\left(\frac{d \psi}{d \omega}\right)_{\omega=1}}{a_{v} k_{f}\left(1+\frac{1}{K}\right)\left(c_{A, b u l k}-\frac{C}{K+1}\right) \frac{4}{3} \pi R_{p}^{3}}
$$

Evaluating the terms and plugging back in to the effectiveness factor yields Equation (A13):

$$
\mathrm{H}=\frac{4 \pi R_{p} D_{\text {eff }}\left(c_{A, b u l k}-\frac{C}{K+1}\right)(\phi \operatorname{coth}(\phi)-1)}{a_{v} k_{f}\left(1+\frac{1}{K}\right)\left(c_{A, b u l k}-\frac{C}{K+1}\right) \frac{4}{3} \pi R_{p}^{3}}=\frac{3 D_{\text {eff }}(\phi \operatorname{coth}(\phi)-1)}{a_{v} k_{f}\left(1+\frac{1}{K}\right) R_{p}^{2}}
$$

Recalling the definition of the Thiele modulus results in Equation (A14):

$$
\mathrm{H}=\frac{3}{\phi^{2}}(\phi \operatorname{coth}(\phi)-1)
$$


Which is the same as the effectiveness factor for the first-order reaction shown in Equation (9) in the main text, with an additional term for the reverse reaction in the potential-dependent portion of the Thiele modulus expression. 


\section{Glossary}

\section{List of Symbols}

Symbol

$a_{c}$

$a_{v}$

C

$c_{A, b u l k}$

$c_{A, \text { surf }}$

$c_{B, \text { bulk }}$

$D_{\text {bulk }}$

$D_{\text {eff }}$

$E_{f}^{0}$

F

$i$

K

$k^{0}$

$k_{f}$

$k_{m}$

$k_{r}$

$L$

$n$

$r$

$R$

$r_{c}$

$R_{p}$

$\bar{r}$

$\bar{r}_{\text {obs }}$

$-$

$r_{\max }$

$\tilde{r}$

$S A$

$T$

$V_{p}$
Description

Exterior volumetric surface area, or shape factor Volumetric surface area

Concentration

Bulk electrolyte concentration of species A

Concentration at the surface of the catalyst

Bulk electrolyte concentration of species B

Molecular diffusivity of reactant species in the bulk electrolyte

Effective reactant diffusivity

Formal potential

Faraday constant

Current density

Dimensionless ratio of forward to reverse rate constant

Standard heterogeneous electrochemical rate constant

Potential-dependent heterogeneous forward rate constant

External mass transfer coefficient

Potential-dependent heterogeneous reverse rate constant

Characteristic length scale

Number of electrons transferred

Coordinate radius

Universal gas constant

Radius of the non-porous core in annulus derivation

Particle radius

Reaction rate flux

Observed reaction rate

Maximum reaction rate

Dimensionless position in annulus analysis

Generic catalyst surface area

Absolute temperature

Generic catalyst volume
Units

$\mathrm{cm}^{-1}$

$\mathrm{cm}^{-1}$

$\mathrm{mol} \mathrm{cm}{ }^{-3}$

$\mathrm{mol} \mathrm{cm}{ }^{-3}$

$\mathrm{mol} \mathrm{cm}{ }^{-3}$

$\mathrm{mol} \mathrm{cm}^{-3}$

$\mathrm{cm}^{2} \mathrm{~s}^{-1}$

$\mathrm{cm}^{2} \mathrm{~s}^{-1}$

$\mathrm{V}$

$\mathrm{C} \mathrm{\textrm {mol } ^ { - 1 }}$

$\mathrm{mA} \mathrm{cm}{ }^{-2}$

$\mathrm{cm} \mathrm{s}^{-1}$

$\mathrm{cm} \mathrm{s}^{-1}$

$\mathrm{cm} \mathrm{s}^{-1}$

$\mathrm{cm} \mathrm{s}^{-1}$

$\mathrm{cm}$

--

$\mathrm{cm}$

$\mathrm{J} \mathrm{mol}^{-1} \mathrm{~K}^{-1}$

$\mathrm{cm}$

$\mathrm{cm}$

$\mathrm{mol} \mathrm{cm}{ }^{-2} \mathrm{~s}^{-1}$

$\mathrm{mol} \mathrm{cm}{ }^{-2} \mathrm{~s}^{-1}$

$\mathrm{mol} \mathrm{cm} \mathrm{cm}^{-2} \mathrm{~s}^{-1}$

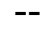

$\mathrm{cm}^{2}$

K

$\mathrm{cm}^{3}$

\section{Greek}

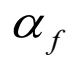

$\alpha_{r}$

$\gamma^{2}$
Forward charge-transfer coefficient

Reverse charge-transfer coefficient

Electrochemically independent Thiele modulus squared
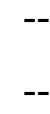
Porosity of catalyst pellet

Overpotential dependent parameter

Overpotential

Effectiveness factor, internal transport limitations only

Effective conductivity

$\mathrm{S} \mathrm{cm}^{-1}$

Ratio of the shell to core thickness

Tortuosity of catalyst pellet

Thiele modulus

Dimensionless concentration using bulk concentration as normalizing basis

Dimensionless position using particle radius as the normalizing basis

Overall effectiveness factor 


\section{References}

1. E. W. Thiele, Industrial \& Engineering Chemistry, 31, 916-920 (1939).

2. O. Levenspiel, Chemical reaction engineering, 3rd ed., p. 668, Wiley, New York, (1999).

3. H. S. Fogler, Elements of Chemical Reaction Engineering, p. 607-625, Prentice Hall, Englewood-Cliffs, (1986).

4. G. Froment, K. Bischoff, and J. De Wilde, Chemical reactor analysis and design, Wiley, New York, (1990).

5. M. E. Davis and R. J. Davis, Fundamentals of Chemical Reaction Engineering, p. 385, Courier Corporation, (2012).

6. J. Pérez-Ramírez, C. H. Christensen, K. Egeblad, C. H. Christensen, and J. C. Groen, Chemical Society Reviews, 37, 2530-2542 (2008).

7. R. T. Driessen, S. R. A. Kersten, and D. W. F. Brilman, Industrial \& Engineering Chemistry Research, 59, 6874-6885 (2020).

8. D. Pletcher and F. C. Walsh, Industrial Electrochemistry, p. 669, Springer Science \& Business Media, (2012).

9. J. Newman and W. Tiedemann, AIChE Journal, 21, 25-41 (1975).

10. R. Zhang, P. He, F. Bai, L. Chen, and W.-Q. Tao, International Journal of Green Energy, 18, 1147-1160 (2021).

11. J. Giner and C. Hunter, J. Electrochem. Soc., 116, 1124 (1969).

12. P. Stonehart and P. N. Ross, Electrochimica Acta, 21, 441-445 (1976).

13. R. P. Iczkowski and M. B. Cutlip, J. Electrochem. Soc., 127, 1433-1440 (1980).

14. M. L. Perry, J. Newman, and E. J. Cairns, J. Electrochem. Soc., 145, 5-15 (1998).

15. T. Duan, J. W. Weidner, and R. E. White, Journal of Power Sources, 107, 24-33 (2002).

16. A. Z. Weber and J. Newman, Chem. Rev., 104, 4679-4726 (2004).

17. W. Sun, B. A. Peppley, and K. Karan, Electrochimica Acta, 50, 3359-3374 (2005).

18. M. Lee, M. Uchida, H. Yano, D. A. Tryk, H. Uchida, and M. Watanabe, Electrochimica Acta, 55, 8504-8512 (2010).

19. X. Zhang, Y. Gao, H. Ostadi, K. Jiang, and R. Chen, Electrochimica Acta, 150, 320-328 (2014). 
20. R. M. Darling, Journal of The Electrochemical Society, 165, F571-F580 (2018).

21. R. Darling, J. Electrochem. Soc., 166, F3058-F3064 (2019).

22. R. M. Darling, J. Electrochem. Soc., 167, 084505 (2020).

23. R. M. Darling and S. F. Burlatsky, J. Electrochem. Soc., 167, 104506 (2020).

24. P. Costamagna, P. Costa, and V. Antonucci, Electrochimica Acta, 43, 375-394 (1998).

25. D. Shin and J. H. Nam, Electrochimica Acta, 171, 1-6 (2015).

26. S. M. Baek, D. Shin, S. Sohn, and J. H. Nam, Fuel Cells, 16, 591-599 (2016).

27. J. H. Nam, J. Electrochem. Sci. Technol, 8, 344-355 (2017).

28. Y. Chen, N. S. Lewis, and C. Xiang, J. Electrochem. Soc., 167, 114503 (2020).

29. J. W. Blake, J. T. Padding, and J. W. Haverkort, Electrochimica Acta, 393, 138987 (2021).

30. J. H. Kim and T. J. Kang, ACS Applied Materials \& Interfaces, 11, 28894-28899 (2019).

31. K. Scott, Electrochimica Acta, 27, 447-451 (1982).

32. K. Scott, Electrochimica Acta, 28, 1191-1200 (1983).

33. B. A. Johnson, A. M. Beiler, B. D. McCarthy, and S. Ott, J. Am. Chem. Soc., 142, 1194111956 (2020).

34. C. Du, X. Liu, G. Ye, X. Gao, Z. Zhuang, P. Li, D. Xiang, X. Li, A. Z. Clayborne, X. Zhou, and W. Chen, ChemSusChem, 12, 1017-1025 (2019).

35. D. Aaron, S. Yeom, K. D. Kihm, Y. Ashraf Gandomi, T. Ertugrul, and M. M. Mench, Journal of Power Sources, 366, 241-248 (2017).

36. M. H. Moghim, R. Eqra, M. Babaiee, M. Zarei-Jelyani, and M. M. Loghavi, Journal of Electroanalytical Chemistry, 789, 67-75 (2017).

37. M. Park, J. Ryu, Y. Kim, and J. Cho, Energy \& Environmental Science, 7, 3727-3735 (2014).

38. Y. Jiang, Y. Li, J. Zhu, Z. He, W. Meng, H. Zhou, L. Wang, and L. Dai, J. Electrochem. Soc., 165, A1813-A1821 (2018).

39. C. T.-C. Wan, D. López Barreiro, A. Forner-Cuenca, J.-W. Barotta, M. J. Hawker, G. Han, H.-C. Loh, A. Masic, D. L. Kaplan, Y.-M. Chiang, F. R. Brushett, F. J. Martin-Martinez, and M. J. Buehler, ACS Sustainable Chem. Eng., 8, 9472-9482 (2020). 
40. A. Forner-Cuenca and F. R. Brushett, Current Opinion in Electrochemistry, 18, 113-122 (2019).

41. A. Z. Weber, M. M. Mench, J. P. Meyers, P. N. Ross, J. T. Gostick, and Q. Liu, J Appl Electrochem, 41, 1137 (2011).

42. Y. Yao, J. Lei, Y. Shi, F. Ai, and Y.-C. Lu, Nature Energy, 1-7 (2021).

43. J. Newman and K. E. Thomas-Alyea, Electrochemical Systems, p. 672, John Wiley \& Sons, (2012).

44. K. Jae Kim, M.-S. Park, Y.-J. Kim, J. Ho Kim, S. Xue Dou, and M. Skyllas-Kazacos, Journal of Materials Chemistry A, 3, 16913-16933 (2015).

45. M. Duduta, B. Ho, V. C. Wood, P. Limthongkul, V. E. Brunini, W. C. Carter, and Y.-M. Chiang, Advanced Energy Materials, 1, 511-516 (2011).

46. Z. Li, K. C. Smith, Y. Dong, N. Baram, F. Y. Fan, J. Xie, P. Limthongkul, W. C. Carter, and Y.-M. Chiang, Phys. Chem. Chem. Phys., 15, 15833-15839 (2013).

47. X. Zhao, B. Jia, Q. Sun, G. Jiao, L. Liu, and D. She, Royal Society Open Science, 5, 180472.

48. B. P. Chaplin, Acc. Chem. Res., 52, 596-604 (2019).

49. H. Dong, Z. Wu, M. J. Liu, and W. A. Tarpeh, Chemical Engineering Journal, 407, 127821 (2021).

50. L. F. Arenas, C. Ponce de León, and F. C. Walsh, Current Opinion in Electrochemistry, 16, 117-126 (2019).

51. V. Presser, C. R. Dennison, J. Campos, K. W. Knehr, E. C. Kumbur, and Y. Gogotsi, Advanced Energy Materials, 2, 895-902 (2012).

52. M. Boota, K. B. Hatzell, E. C. Kumbur, and Y. Gogotsi, ChemSusChem, 8, 835-843 (2015).

53. T. Tomai, H. Saito, and I. Honma, J. Mater. Chem. A, 5, 2188-2194 (2017).

54. W. M. Deen, Analysis of Transport Phenomena, (1998).

55. B. Corry, S. Kuyucak, and S. H. Chung, Chemical Physics Letters, 320, 35-41 (2000).

56. H. Daiguji, Chem. Soc. Rev., 39, 901-911 (2010).

57. R. M. Darling and M. L. Perry, J. Electrochem. Soc., 161, A1381-A1387 (2014).

58. J. D. Milshtein, K. M. Tenny, J. L. Barton, J. Drake, R. M. Darling, and F. R. Brushett, J. Electrochem. Soc., 164, E3265-E3275 (2017). 
59. M. Secanell, K. Karan, A. Suleman, and N. Djilali, Electrochimica Acta, 52, 6318-6337 (2007).

60. V. Ramadesigan, R. N. Methekar, F. Latinwo, R. D. Braatz, and V. R. Subramanian, J. Electrochem. Soc., 157, A1328 (2010).

61. J. W. Haverkort, Electrochimica Acta, 295, 846-860 (2019).

62. T. F. Fuller and J. N. Harb, Electrochemical Engineering, p. 450, John Wiley \& Sons, (2018).

63. R. G. Compton and C. E. Banks, Understanding Voltammetry (Second Edition), p. 429, World Scientific, (2011).

64. S. Zhong and M. Skyllas-Kazacos, Journal of Power Sources, 39, 1-9 (1992).

65. A. Alazmi, O. E. Tall, M. N. Hedhili, and P. M. F. J. Costa, Inorganica Chimica Acta, 482, 470-477 (2018).

66. https://www.fuelcellstore.com/spec-sheets/vulcan-xc72-spec-sheet.pdf.

67.

http://www.mtixtl.com/TIMCALGraphiteandCarbonSuperPConductiveCarbonBlack100g/bagEQ-Li.aspx.

68. R. Aris, Chemical Engineering Science, 6, 262-268 (1957).

69. K. Amini, J. Gostick, and M. D. Pritzker, Advanced Functional Materials, 30, 1910564 (2020).

70. M. H. Gharahcheshmeh, C. T.-C. Wan, Y. A. Gandomi, K. V. Greco, A. Forner-Cuenca, Y.M. Chiang, F. R. Brushett, and K. K. Gleason, Advanced Materials Interfaces, 7, 2000855 (2020).

71. D.-S. Yang, J. H. Han, J. W. Jeon, J. Y. Lee, D.-G. Kim, D. H. Seo, B. G. Kim, T.-H. Kim, and Y. T. Hong, Materials Today Energy, 11, 159-165 (2019).

72. X. Zhang, Q. Wu, Y. Lv, Y. Li, and X. Zhou, Journal of Materials Chemistry A, 7, 2513225141 (2019).

73. J. Sun, H. R. Jiang, M. C. Wu, X. Z. Fan, C. Y. H. Chao, and T. S. Zhao, Applied Energy, 271, 115235 (2020).

74. B. Sun and M. Skyllas-Kazacos, Electrochimica Acta, 37, 1253-1260 (1992).

75. K. V. Greco, A. Forner-Cuenca, A. Mularczyk, J. Eller, and F. R. Brushett, ACS Appl. Mater. Interfaces, 10, 44430-44442 (2018).

76. B. Sun and M. Skyllas-Kazacos, Electrochimica Acta, 37, 2459-2465 (1992). 
77. Y. Men and T. Sun, Int. J. Electrochem. Sci., 7, 7 (2012).

78. D. K. Stevens, P. M. Berthouex, and T. W. Chapman, Journal of Environmental Engineering, 113, 1149-1155 (1987).

79. J. P. Lopes, S. S. S. Cardoso, and A. E. Rodrigues, Chemical Engineering Science, 71, 46-55 (2012). 